\title{
Protein charge parameters that influence stability and cellular internalization of polyelectrolyte complex micelles
}

\author{
Rachel A. Kapelner, Allie C. Obermeyer* \\ Department of Chemical Engineering, Columbia University, New York, NY 10027
}

\begin{abstract}
Proteins are an important class of biologics, but there are several recurring challenges to address when designing protein-based therapeutics. These challenges include: the propensity of proteins to aggregate during formulation, relatively low loading in traditional hydrophobic delivery

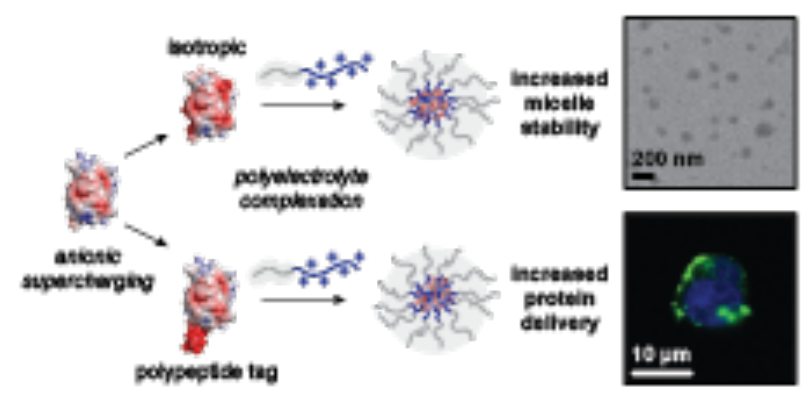
vehicles, and inefficient cellular uptake. This last criterion is particularly challenging for anionic proteins as they cannot cross the anionic plasma membrane. Here we investigated the complex coacervation of anionic proteins with a block copolymer of opposite charge to form polyelectrolyte complex (PEC) micelles for use as a protein delivery vehicle. Using genetically modified variants of the model protein green fluorescent protein (GFP), we evaluated the role of protein charge and charge localization in the formation and stability of PEC micelles. A neutralcationic block copolymer, POEGMA 79 -b-qP4VP ${ }_{175}$, was prepared via RAFT polymerization for complexation and microphase separation with the panel of engineered anionic GFPs. We found that isotropically supercharged proteins formed micelles at higher ionic strength relative to protein variants with charge localized to a polypeptide tag. We then studied GFP delivery by PEC micelles and found that they effectively delivered the protein cargo to mammalian cells. However, cellular delivery varied as a function of protein charge and charge distribution and we found an inverse relationship between the PEC micelle critical salt concentration and delivery efficiency. This model system has highlighted the potential of polyelectrolyte-complexes to deliver anionic proteins intracellularly as well as the importance of correlating solution structure and desired functional activity.
\end{abstract}




\section{Introduction}

Advances in genetic engineering and biotechnology have provided the ability to design, develop, and produce proteins as therapeutic treatments. To date, hundreds of protein therapeutics have been developed and approved by the FDA. However, currently approved protein therapies act primarily on extracellular or membrane-bound targets. ${ }^{1}$ This is largely due to the inability of proteins to cross the cell membrane and access intracellular targets. ${ }^{1-3}$ To fully realize the promise of protein therapeutics, efficient intracellular protein delivery is required. Intracellular delivery of a protein molecule, as opposed to the cDNA or mRNA for the cell to produce the protein itself, ensures the best control over dosage. ${ }^{4}$ Current methods of protein delivery have a few key shortcomings including inefficient cellular uptake and poor endosomal escape. Therefore, to capitalize on the potential of genetic and protein engineering to treat disease, new methods for intracellular protein delivery are needed. ${ }^{3,4}$

Two major strategies have been studied to improve intracellular protein delivery: increasing the hydrophobicity of the protein and increasing the cationic charge on the protein. ${ }^{5}$ It has previously been demonstrated that hydrophobic proteins can translocate across the cell membrane, most likely due to favorable hydrophobic interactions with the lipid bilayer. Mix et al. were able to deliver an esterified green fluorescent protein (GFP) directly to the cytosol. ${ }^{6}$ Esterification of the carboxyl groups with a hydrophobic diazo compound was sufficient for the protein to directly cross the cellular membrane. Additionally, hydrophobic cell-penetrating peptides (CPPs) have been shown to deliver GFP to HeLa cells. ${ }^{7}$ Cationic CPPs, particularly peptides with a high fraction of arginine residues, have been shown to similarly facilitate intracellular protein delivery. ${ }^{8-11}$ Similarly, proteins with significant cationic charge, native or engineered, can also be internalized efficiently by cells. ${ }^{12,13}$

For anionic proteins to cross the cellular membrane they must overcome the Coulombic repulsion from the negatively charged extracellular membrane ${ }^{14}$ and the hydrophobic nature of the lipid bilayer. ${ }^{6}$ It is for this reason that anionic proteins remain a particular challenge to deliver intracellularly. Yet at the same time, the delivery of highly anionic nucleic acids has been successfully demonstrated and implemented. Several approaches to deliver anionic proteins has taken inspiration from DNA delivery approaches. These include the formulation of protein nanoparticles, ${ }^{15-18}$ polymer nanoemulsions, ${ }^{19,20}$ and liposomes. ${ }^{21-23}$ While all have 
demonstrated successes, these techniques tend to be better suited for nucleic acid delivery due to the high charge density of nucleic acids.

An alternative approach for protein delivery uses polyelectrolyte complex (PEC) micelles formed between a protein and diblock copolymer. This approach still looks to DNA delivery for inspiration, as PEC micelles were initially developed by Kataoka et al. for nucleic acid delivery in the late 1990's. ${ }^{24-27}$ These PEC micelles are formed from the electrostatic attraction between a diblock copolymer with a charged block and a hydrophilic charge-neutral block and a protein of opposite charge. ${ }^{28}$ Encapsulating supernegatively charged proteins in the core of a PEC micelle could potentially serve to shield the protein from enzymatic degradation prior to delivery, promote intracellular delivery of the protein, and even facilitate endosomal escape. ${ }^{29}$

PEC micelles have been demonstrated to successfully deliver nucleic acids, but because proteins have a lower charge density, initial studies have shown that at physiological ionic strength, protein-based PEC micelles dissociate. ${ }^{30,31}$ To overcome this challenge, previous work has explored different ways to increase the charge density of the PEC micelle core. One strategy is to introduce a third polyelectrolyte, which would be co-encapsulated in the micelle core with the like-charged protein. While this strategy has been shown to increase the salt stability of the micelle, the protein component partitioned out of the micelle below the micelle critical salt concentration, rendering this approach infeasible for applications at physiological conditions. ${ }^{32,33}$ Another strategy is to increase the net charge of the protein itself. Increasing the net negative charge of four model proteins, $\alpha$-chymotrypsinogen, lysozyme, myoglobin, and RNase A, by chemical supercharging was shown to increase the salt stability of PEC micelles. ${ }^{34}$ Similarly, supercharging equine heart cytochrome $c(\mathrm{CytC})$ and immunoglobulin $\mathrm{G}(\mathrm{IgG})$ with citraconic anhydride or cis-aconitic anhydride resulted in the formation of PEC micelles that remained stable at physiological ionic strength..$^{29,35,36}$ The reversible nature of the chemical supercharging of proteins in this approach had the added benefit of promoting $\mathrm{pH}$ responsive PEC micelle dissolution and endosomal escape in model mammalian cell lines.

While chemical supercharging a protein has produced salt stable PEC micelles for intracellular delivery, there are a few drawbacks to this strategy. Chemical supercharging results in a distribution of supercharged proteins, with polydisperse charge densities; the behavior of the resultant proteins is dependent on reaction conditions and degrees of supercharging, with high 
degrees of supercharging frequently required for improved delivery efficiency ${ }^{36}$ Additionally, many proteins are not stable or catalytically active at the degree of modification required for saltstable micelle formation. Genetic engineering enables the selection of the location of each charged residue on the protein surface and a monodisperse distribution of net charge. Based on previous work on complex coacervation with homopolymers, protein charge distribution can also impact the conditions where phase separation is observed. ${ }^{37-42}$ To date, both the effect of the degree of protein supercharging and charge distribution on PEC micelle formation and stability has yet to be established. In this work, genetic engineering was used to precisely control the number and location of charges on a model protein, GFP. A relationship between protein net charge and charge distribution on micelle stability was established and, for isotropically charged proteins, this relationship was consistent with the behavior of analogous bulk-phase coacervates. In addition, the effect of PEC micelle stability on intracellular delivery efficiency was assessed.

\section{Results and discussion}

\section{Design of protein-polyelectrolyte delivery system.}

Given the inability of anionic proteins to enter cells, we designed a polymer delivery vehicle to improve intracellular delivery (Scheme 1a). This approach relies on complexation and microphase separation of anionic proteins with a cationic block copolymer. At appropriate solution conditions, mixing GFP with this block copolymer results in the formation of spherical polyelectrolyte complex (PEC) micelles with a GFP-rich core. However, in order to effectively deliver proteins using this approach, an understanding of how protein properties impact the formation and stability of PEC micelles is needed. To assess the effect of protein charge on PEC micelle formation and stability, we generated a library of negatively charged superfolder GFP (sfGFP) mutants (Scheme 1b). We have previously demonstrated the relationship between protein charge and both the nature of phase separation (solid or liquid) and the phase behavior at increased ionic strength. ${ }^{37}$ Based on these prior findings and the requirement for phase separation at physiological ionic strength, only GFP variants with higher net charge were investigated. A panel of GFP mutants with an expected charge of $-18,-24$, and -30 were evaluated. For each of these variants, the charge was distributed isotropically across the protein surface. 
a

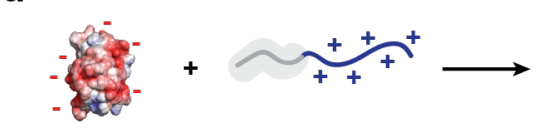

supercharged POEGMA-b-qP4VP

b GFP
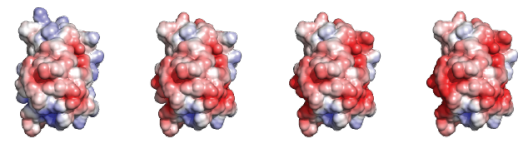

$\operatorname{GFP}(-7)$

GFP(-18) GFP(-24) GFP(-30)

C
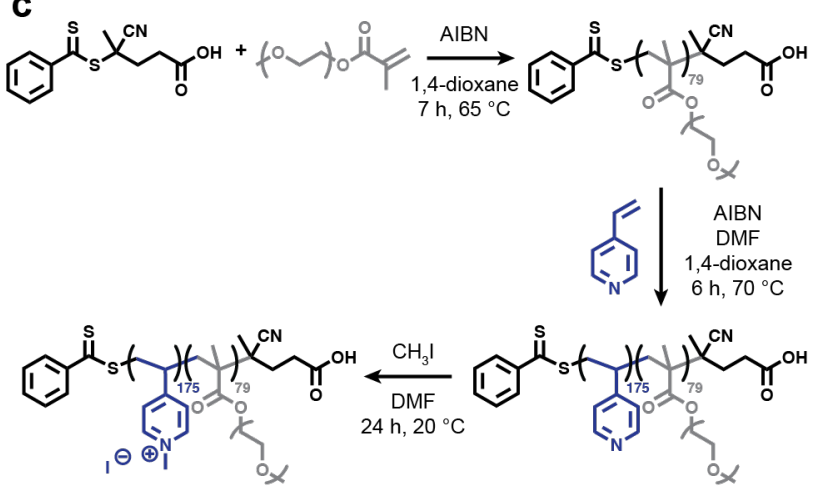

Scheme 1. (a) Schematic of polyelectrolyte core (PEC) micelle formation. (b) Electrostatic maps of GFP(-7) (superfolder) and supercharged GFP variants studied here. (c) Synthesis of the diblock copolymer, POEGMA $A_{70}-b-q P 4 V P_{175}$, via RAFT polymerization. The chain transfer agent (CTA) used was 4-cyano-4-(phenylcarbonothioylthio)pentanoic acid, the initiator used was azobisisobutyronitrile (AIBN), and the OEGMA monomer used had a $M_{n}=300 \mathrm{~g} / \mathrm{mol}(\mathrm{n} \sim 4-5)$.

Variants with equivalent charge localized to a Cterminal polypeptide tag were also generated. The addition of glutamate and aspartate residues at the C-terminus of GFP generated variants with a polyionic tag that provided a localized charge patch for polyelectrolyte complexation. The tagged variants with net charge of -18 and -24 were successfully characterized, but insufficient quantity of pure tag-GFP(-30), with 24 charged residues localized to the $\mathrm{C}$-terminus, was produced for characterization (Supporting Figure S1). Finally, the unmodified sfGFP protein was also characterized for comparison to the globular domain of the tagged variants. The hydrodynamic radius of purified proteins was characterized by DLS. As expected, all characterized proteins had a hydrodynamic radius of approximately $2 \mathrm{~nm}$, with the exception of GFP(-18) (Supporting Figure S1). GFP(-18) had a monomeric population with an average radius of $2 \mathrm{~nm}$, but also had a population with a larger radius $(\sim 11$ $\mathrm{nm}$ ). This larger population has been interpreted as protein multimers, which is consistent with the ability of sfGFP to form a weak dimer. ${ }^{43}$

We then proceeded to design a block copolymer that could complex these anionic proteins and form PEC micelles (Scheme 1c). A neutral-cationic diblock polymer was designed with a poly(oligo(ethylene glycol) methyl ether methacrylate) (POEGMA) charge neutral block and a quaternized poly(4-vinyl- $N$-methylpyridinium) (qP4VP) cationic block. POEGMA was chosen for the neutral corona block, due to demonstrated cytocompatibility and its comb-like architecture, which has been shown to increase cellular uptake over linear poly(ethylene glycol).44,45 The strong polycation, qP4VP, was selected for the cationic block because previous work had carefully characterized the phase behavior of these anionic GFPs with a qP4VP homopolymer. ${ }^{37}$ To prepare the block copolymer, the POEGMA block was first synthesized by reversible addition- 
fragmentation chain-transfer (RAFT) polymerization. The molecular weight of the POEGMA block, $M_{n}=23.7 \mathrm{~kg} \mathrm{~mol}^{-1}$, was determined by gel permeation chromatography (Supporting Figure S2). Subsequently, the poly(4-vinylpyridine) (P4VP) block was synthesized via RAFT polymerization, using the POEGMA block as a macromolecular chain transfer agent. The resulting block copolymer had a block ratio with 2.2 4VP monomers to 1 OEGMA monomer, as determined by ${ }^{1} \mathrm{H}$ NMR (Supporting Figure $\mathrm{S} 3$ ). The P4VP block was quaternized ( 95\%) with an excess of methyl iodide, resulting in the final block copolymer, POEGMA $79-b$-qP4VP 175 (Supporting Figure S3).

\section{Polyelectrolyte complex (PEC) micelle formation.}

Micelle formation was initially studied by evaluating several protein and block copolymer mixing ratios using dynamic light scattering (DLS) in the absence of salt (Figure 1a, Supporting Figure S4). For these experiments, the overall macromolecule concentration was kept constant at $0.2 \mathrm{mg} \mathrm{mL}^{-1}$. All of the mutants formed micelles at several mixing ratios. Our first observation was that PEC micelles form at positive charge fractions, $f^{+}$, greater than 0.5. Because polyelectrolyte complex micelle formation is driven by polyelectrolyte complexation, we would expect micelle formation to be most favorable at charge neutrality or $f^{+}=0.5$. We hypothesize that this shift to higher charge fractions, which indicates that phase separation is favored in the
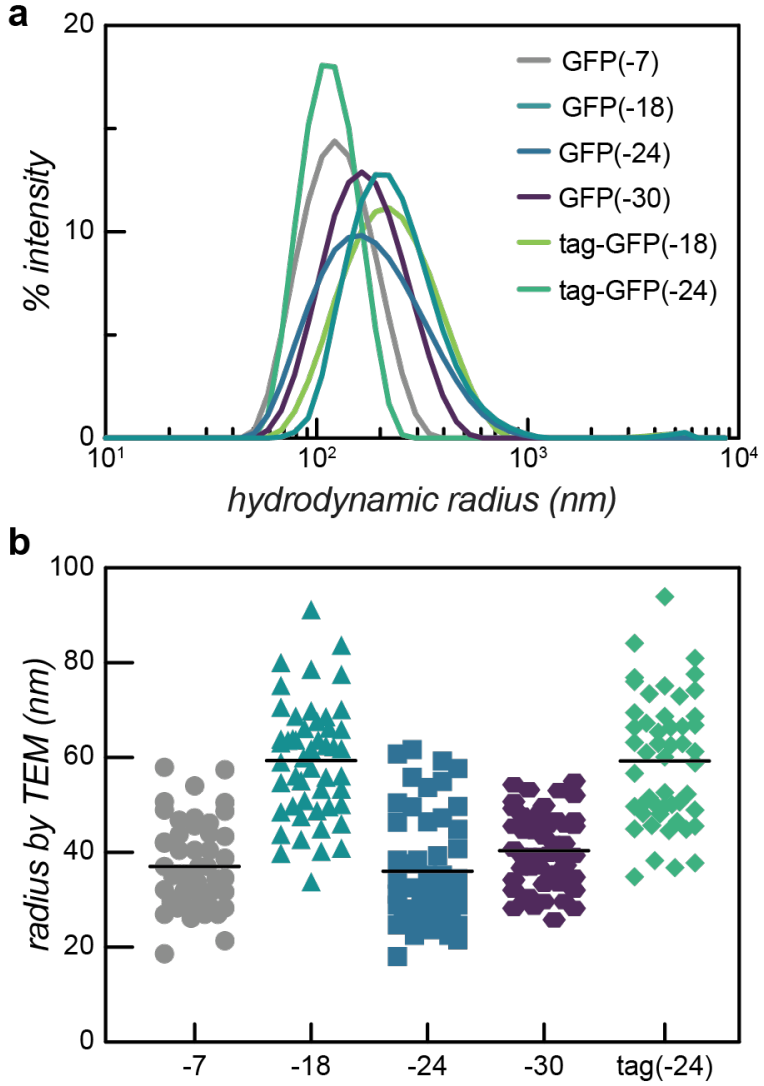

c

GFP(-7)
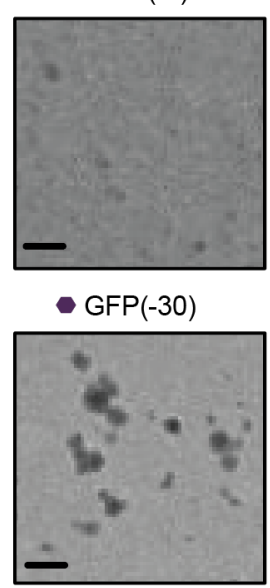

Figure 1. Effect of protein charge on the formation of polyelectrolyte complex micelles (PECMs). (a) Representative DLS micelle intensity traces for samples prepared with $0.1 \mathrm{mg} \mathrm{mL}^{-1}$ protein and $0.1 \mathrm{mg} \mathrm{mL}^{-1}$ polymer in $10 \mathrm{mM}$ tris, $\mathrm{pH} 7.4$. Traces are an average of 3 samples measured $1 \mathrm{~h}$ after mixing. (b) Micelle radius as measured by TEM, $\mathrm{n}=50$. The black bar indicates the mean. (c) TEM micrographs of PECMs prepared with $0.05 \mathrm{mg} \mathrm{mL}^{-1}$ protein and $0.05 \mathrm{mg} \mathrm{mL}^{-1}$, with the exception of GFP(-7) and tag-GFP(-24) which were prepared with $0.1 \mathrm{mg} \mathrm{mL}^{-1}$ protein and $0.1 \mathrm{mg} \mathrm{mL}^{-1}$. Scale bar $=200 \mathrm{~nm}$. 
presence of excess positive charge, is due to the ionizable nature of proteins. Upon complexation with the polycation, ionizable side chains (His, Lys, Arg) can alter protonation state, making the protein more net negatively charged. Upon charge regulation, fewer proteins are required per polymer chain to achieve charge neutrality. Interestingly, for the isotropic supercharged variants, the charge fraction that resulted in the maximum derived count rate for each mutant decreased with decreasing protein net charge. The derived count rate is correlated with the DLS scattering intensity and is indicative of higher concentration or larger particles. While potentially counterintuitive, as this implies that fewer of the less negatively charged mutants are required to neutralize the polymer, this is also consistent with previous observations of anionic GFP macrophase separation. ${ }^{37}$ We hypothesize that for less negatively charged proteins, there are more opportunities for induced charging in the presence of the polycation, resulting in a larger deviation from the hypothetical charge neutral mixing ratio. In addition to the optimal mixing ratio depending on protein charge, the charge distribution also impacted micelle formation as a function of mixing ratio. For example, tag-GFP(-24) formed PEC micelles over fewer mixing ratios than GFP(-24) (Supporting Figure S4). This is also consistent with previous findings, where phase separation of proteins with polyionic tags was observed for fewer mixing ratios than their isotropically charged analogs. We hypothesize this behavior can be attributed to either differences in protein geometry or the comparatively lower average distance between like charges for the tagged variants.

The formation of spherical micelles was confirmed by transmission electron microscopy (TEM, Figure 1b,c, Supporting Table S2) for most of the GFP variants. These GFP variants formed spherical micelles in $10 \mathrm{mM}$ tris with $0 \mathrm{mM} \mathrm{NaCl}$. Micelles formed from tag-GFP(-18) could not be visualized by TEM. Clusters of several spherical particles were observed for some of the isotropic variants and were excluded from subsequent particle size analysis. The hydrodynamic radius determined by TEM was smaller than that determined by DLS. We hypothesize this discrepancy is a result of only the core of the micelle having sufficient electron density to generate contrast in TEM. ${ }^{46,47}$ For the isotropic supercharged variants, the least negatively charged protein, GFP(-18), had the largest radius both by TEM and DLS. One hypothesis for this observation is that PEC micelles formed with the less negatively charged GFP mutant would require more protein molecules in the core to achieve charge neutrality, resulting in a larger core. This is consistent with previous research with lipase, where a positive correlation between the 
number of lipase molecules in the core of the micelle and the size of the PEC micelle core was observed. ${ }^{33}$ The other two isotropic variants showed similar radii by both measurements. In contract, for the tagged GFP variant, tag-GFP(-24), the radii measured by DLS and TEM were nearly identical. We hypothesize this was due to the swelling of these micelles upon dilution for TEM analysis. This swelling in the absence of salt was only observed for tag-GFP(-24), most likely due to the lower salt stability of this variant, as described below.

\section{PEC micelle stability.}

With the addition of small amounts of $\mathrm{NaCl}(25 \mathrm{mM})$, the hydrodynamic radius of PEC micelles formed with isotropically supercharged GFPs decreased (Figure 2, Table 1, Supporting Table S3). By DLS, micelles formed with GFP(-18) decreased by $\sim 30 \%$ and those formed by GFP(24 ) and GFP( $(-30)$ deceased by $\sim 25 \%$ with the addition of $25 \mathrm{mM} \mathrm{NaCl}$. However, this decrease

a

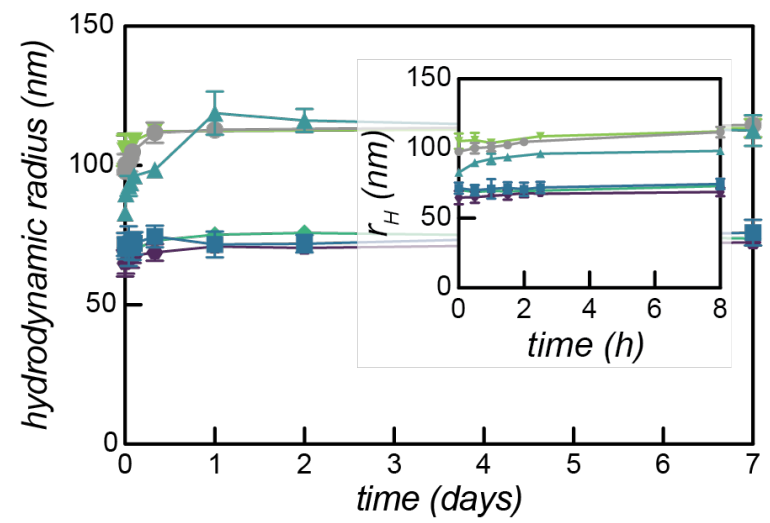

C

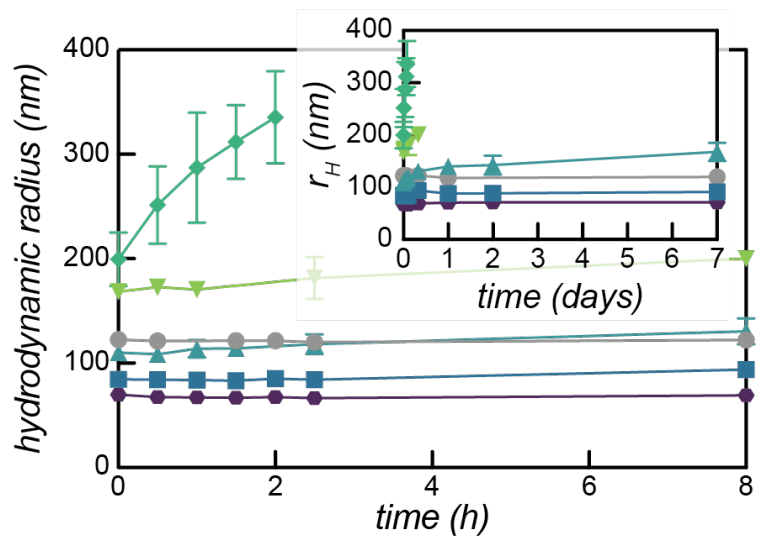

b

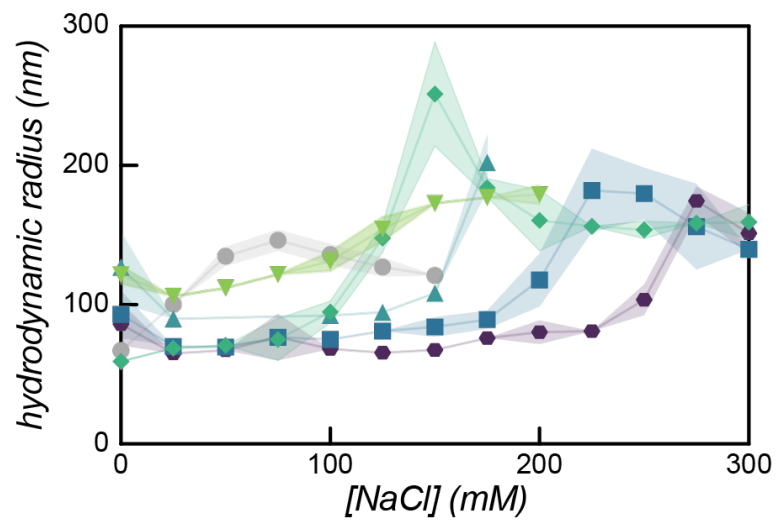

d
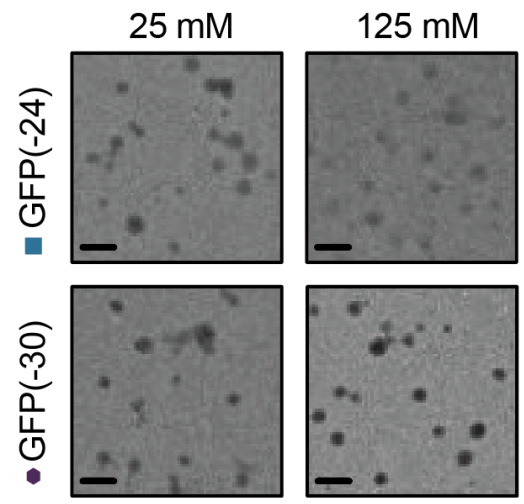

$150 \mathrm{mM}$

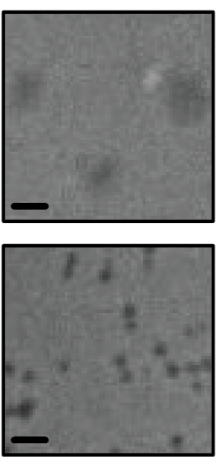

- GFP(-7) $\triangle$ GFP(-18)

GFP(-24)

- GFP(-30)

$\checkmark$ tag-GFP(-18)

tag-GFP(-24)

Figure 2. PECM stability as a function of protein charge. (a) Temporal stability of micelles was evaluated using the hydrodynamic radius of the DLS intensity of peak 1 over time. Samples were prepared with $0.1 \mathrm{mg} \mathrm{mL}^{-1}$ protein and $0.1 \mathrm{mg} \mathrm{mL}^{-1}$ polymer in $10 \mathrm{mM}$ tris, $\mathrm{pH} 7.4 \mathrm{with} 25 \mathrm{mM}$ NaCl. (b) Salt stability as measured by the hydrodynamic radius determined from the DLS intensity of peak 1 . The shaded area corresponds to the standard deviation of three repliactes. Samples were prepared with $0.1 \mathrm{mg} \mathrm{mL}^{-1}$ protein and $0.1 \mathrm{mg} \mathrm{mL}^{-1}$ polymer in $10 \mathrm{mM}$ tris, $\mathrm{pH} 7.4$ with varying concentrations of NaCl. (c) Temporal stability of the PECMs was also evaluated at physiological ionic strength (150 mM NaCl) as in (a). (d) TEM micrographs of GFP(-24) and GFP(-30) based PECMs prepared at different salt concentrations. Samples were prepared with $0.05 \mathrm{mg} \mathrm{mL}^{-1}$ protein and $0.05 \mathrm{mg} \mathrm{mL}^{-1}$ at 25,125 , and $150 \mathrm{mM} \mathrm{NaCl}$. Scale bar $=200 \mathrm{~nm}$. 
in size is not reflected in the radius measured by TEM (Table 1). This indicates that while the small addition of salt does not cause the core to swell, it might alter the nature of the phase separation in the core. Increasing the system ionic strength would make the core either more liquid-like, if it was solid or gel-like at $0 \mathrm{M} \mathrm{NaCl}$, or decrease the viscosity of a liquid core, resulting in a PEC micelle population with fewer kinetically trapped aggregates and an overall narrower particle size distribution. This was reflected in the decrease in polydispersity index (PDI) as measured by DLS (Supporting Tables S2-3). The PDI of the PEC micelles formed in $25 \mathrm{mM}$ $\mathrm{NaCl}$ was half that of the PEC micelle solutions with no salt. The decrease in the standard deviation of the intensity peaks further reflect that the addition of $25 \mathrm{mM} \mathrm{NaCl}$ results in a more homogeneous particle population.

In contrast, the hydrodynamic radius of micelles formed with a tagged GFP variant, tag-GFP(24 ), increased by $\sim 15 \%$ under the same conditions and the PDI did not decrease with the addition of a small amount of salt. We hypothesize that the PEC micelles formed with this tagged variant resulted in a liquid-like core with a lower viscosity than PEC micelles formed with isotropic variants. This is based on previous findings that demonstrated that tagged GFP variants were more likely to form liquid complex coacervates with a qP4VP homopolymer. ${ }^{37}$ Isotropic GFP variants formed solid precipitates with the same qP4VP homopolymer. While the lower macromolecule concentrations used in this study likely prevents the formation of solid complexes, as indicated by the spherical nature of the PEC micelles at low salt, we propose that the addition of salt to this PEC micelle system decreases the viscosity of the core, enabling more rapid chain rearrangement and exchange. This results in smaller PEC micelle hydrodynamic radii with the isotropic mutants at low salt concentrations. However, the lower net charge tagged variant, tag-GFP(-18), showed similar behavior to the isotropic variants with both a decrease in micelle size and PDI upon the addition of $25 \mathrm{mM} \mathrm{NaCl}$. This variant had a derived count rate that was nearly an order of magnitude lower than the other variants, indicating limited formation and stability of PEC micelles. We attribute the different behavior of tag-GFP(-18) to the relatively poor formation of micelles even in the absence of salt.

The colloidal stability of the micelles at low salt was monitored for 7 days by DLS (Figure 2a). In addition to all GFP variants forming PEC micelles at low salt, all mutants formed micelles that remained colloidally stable for 7 days in the presence of $25 \mathrm{mM} \mathrm{NaCl}$. Under these conditions, 
minimal micelle swelling was observed in the first $8 \mathrm{~h}$ for tag-GFP(-18) $(+7 \pm 5 \%)$, tag-GFP(-24) $(+4 \pm 3 \%)$, GFP(-24) $(+4 \pm 8 \%)$, and GFP(-30) (+6 $\pm 9 \%)$. On the other hand, in $25 \mathrm{mM} \mathrm{NaCl}$ GFP(-18) micelles swelled $+19 \pm 1 \%$ in the first $8 \mathrm{~h}$ and were $+43 \pm 7 \%$ larger than the original size by $24 \mathrm{~h}$. Following this initial swelling, GFP(-18) micelles remained stable at this size for the remainder of the week. Interestingly, this swollen micelle size for GFP(-18) in $25 \mathrm{mM} \mathrm{NaCl}$ was similar to the micelle size in the absence of $\mathrm{NaCl}(117 \pm 12 \mathrm{~nm}$ at $\mathrm{t}=0$ in $0 \mathrm{mM} \mathrm{NaCl}$ and $119 \pm$ $8 \mathrm{~nm}$ at $\mathrm{t}=24 \mathrm{~h}$ in $25 \mathrm{mM} \mathrm{NaCl})$. This swelling behavior of $\operatorname{GFP}(-18)$ micelles at modest ionic strength was observed at non-equal mass mixing ratios as well (Supporting Figure S5). In addition to changes in micelle size, the derived count rate for $\operatorname{GFP}(-18)$ micelles also indicated changes in temporal stability at $25 \mathrm{mM} \mathrm{NaCl}$ (Supporting Figure S6). Without the addition of salt, the micelles maintained a constant derived count rate over 7 days. But, with the addition of 25 $\mathrm{mM} \mathrm{NaCl}$, micelles formed with GFP(-18) saw an initial increase in the derived count rate over the first $24 \mathrm{~h}$, but then had a stable count rate for the next 6 days, again indicative of an increased particle size in solution.

After establishing the colloidal stability at low salt, micelle formation and stability as a function of ionic strength was assessed (Figure 2b). Mixtures of protein and polymer at varying ionic strengths were prepared by first mixing protein solutions with different quantities of a $5 \mathrm{M} \mathrm{NaCl}$

Table 1. Summary of PEC micelle data for protein panel

\begin{tabular}{|c|c|c|c|c|c|c|c|}
\hline \multirow[t]{2}{*}{ Protein } & \multirow[t]{2}{*}{$f_{+}^{+}$} & \multirow[t]{2}{*}{ Protein Fraction } & $\mathrm{r}_{\mathrm{H}}, \mathrm{nm}, \mathrm{DLS}$ & $r_{H}, \mathrm{~nm}, \mathrm{TEM}$ & $\mathrm{r}_{\mathrm{H}}, \mathrm{nm}, \mathrm{DLS}$ & $\mathrm{r}_{\mathrm{H}}, \mathrm{nm}, \mathrm{TEM}$ & \multirow[t]{2}{*}{$\begin{array}{c}\text { Critical Salt } \\
\text { Concentration, } \\
\mathrm{mM}\end{array}$} \\
\hline & & & \multicolumn{2}{|c|}{$0 \mathrm{mM} \mathrm{NaCl}$} & \multicolumn{2}{|c|}{$25 \mathrm{mM} \mathrm{NaCl}$} & \\
\hline $\operatorname{sfGFP}(-7)$ & 0.91 & 0.5 & $67 \pm 1$ & $37 \pm 9$ & $98 \pm 3$ & - & 25 \\
\hline \multirow{2}{*}{ GFP(-18) } & 0.85 & 0.4 & $90 \pm 4$ & & $68 \pm 2$ & $59 \pm 15$ & 125 \\
\hline & 0.79 & 0.5 & $117 \pm 12$ & $57 \pm 13$ & $83 \pm 1$ & $59 \pm 12$ & 125 \\
\hline GFP(-24) & 0.73 & 0.5 & $101 \pm 12$ & $36 \pm 12$ & $72 \pm 4$ & $45 \pm 11$ & 175 \\
\hline GFP(-30) & 0.69 & 0.5 & $92 \pm 14$ & $40 \pm 8$ & $65 \pm 5$ & $59 \pm 13$ & 225 \\
\hline tag-GFP(-18) & 0.79 & 0.5 & $119 \pm 7$ & - & $105 \pm 6$ & - & 75 \\
\hline tag-GFP(-24) & 0.74 & 0.5 & $60 \pm 1$ & $59 \pm 13$ & $70 \pm 2$ & - & 100 \\
\hline
\end{tabular}

solution, followed by addition of the polymer solution. Shortly after mixing, the particles in solution were monitored by DLS. Critical salt concentrations were established by the observed swelling of the hydrodynamic radius as a function of salt concentration (Figure $2 b$, Table 1). These observed increases in hydrodynamic radius also corresponded with a decrease in the derived 
count rate, indicating a decrease in the scattering intensity of the solution (Supporting Figure S7).

The isotropically charged mutants demonstrated a clear trend with increasing negative charge improving the micelle salt stability (Table 1). As the magnitude of protein charge increases, so does the number of potential interaction sites with the polycation, resulting in an increase in the overall strength of interaction between the polymer and protein. Therefore, a higher salt concentration was required for microphase separation to no longer be entropically favorable. In comparison, we observed that micelles formed from tagged GFP variants had lower critical micelle salt concentrations. We hypothesize that this is due to differences between the interactions of the globular domain with the qP4VP block and the polyionic tag with the qP4VP

a

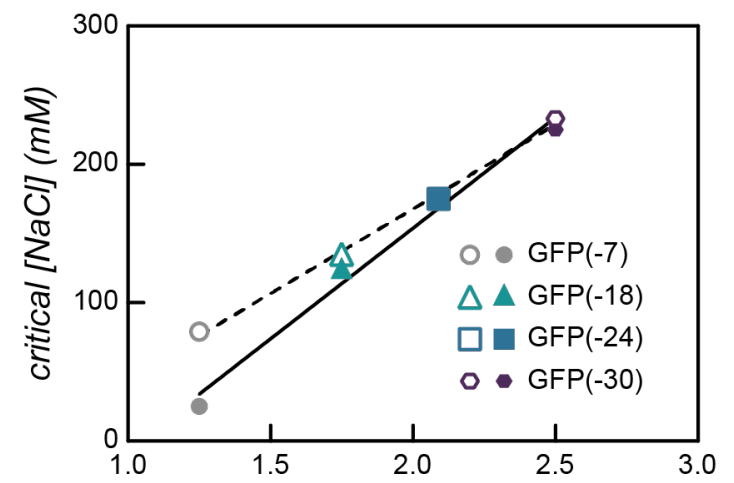

b

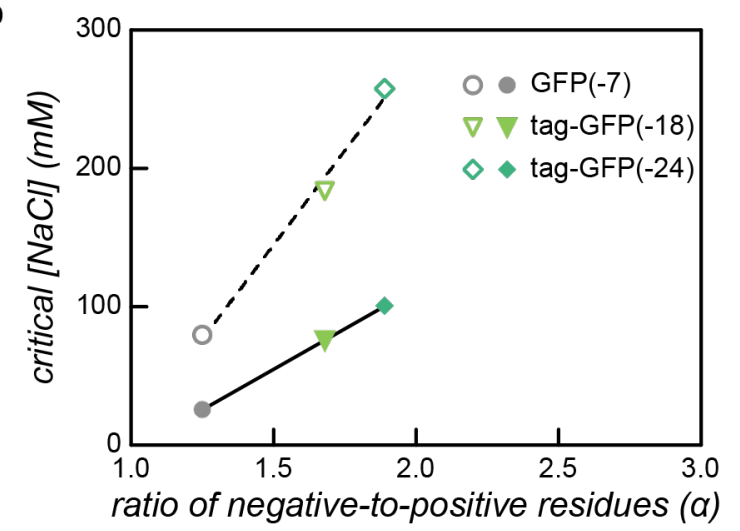

Figure 3. Relationship between critical salt concentration and the ratio of negative to positive residues on the protein. (a) For isotropically supercharged variants, the critical salt concentration for macro- (open symbols, dashed line) and microphase separation (filled symbols, solid line) are nearly identical. (b) For GFP variants that were supercharged via a polyionic peptide tag, the critical salt concentration for microphase separation (filled symbols, solid line) is similar to the critical salt concentration for macrophase separation of the globular domain (GFP(-7), grey open circle). block. It has previously been demonstrated that all of these GFP mutants remain phase separated with a qP4VP homopolymer at physiological ionic strength, albeit at a 5-fold higher macromolecule concentration. ${ }^{37}$ The salt stability data for macrophase separation indicates that the tag interacts more strongly with qP4VP than the isotropic equivalent. This resulted in a comparatively higher critical salt concentrations for tagged GFPs with the qP4VP homopolymer than the isotropically charged counterparts (Figure 3). In contrast, for PEC micelles the isotropically supercharged GFP micelles remain assembled at higher salt concentrations than the tagged GFP variants. However, while micelles formed with a tagged variant, tag-GFP(-24), swelled and then disassembled above $100 \mathrm{mM} \mathrm{NaCl}$, particles with a hydrodynamic radius slightly larger than 
the free protein ( $\sim 7 \mathrm{~nm}$ particles; $\sim 2 \mathrm{~nm}$ protein) were observed via the DLS number distribution at higher salt concentrations (Supporting Figure S8). This behavior was not observed for GFP(24) PEC micelles. We hypothesize that the increasing salt concentration preferentially screens the interactions between the polymer and the protein globular domain, which has an expected charge of -7 , but not between the polymer and the charged tag, which has an expected charge of -18. Polymer interactions with both the tag and globular domain would allow more than one polymer chain to interact with each protein within the PEC micelle core. Screening the polymerglobular domain interactions could result in PEC micelle swelling and dissociation. Given that the tagged variants have the least negative globular domain, we would then expect the critical micelle salt concentration of the tagged variants to be the lowest. But concurrently, the tag domain could still facilitate interactions with the polymer above the critical micelle salt concentration but below the critical salt concentration established with the qP4VP homopolymer. This could result in the formation of smaller protein-polymer complexes with one polymer molecule complexed with a few proteins, indicated by the $\sim 7 \mathrm{~nm}$ particle size that is observed by DLS. 48,49

To better understand the particle populations present during protein delivery applications, the temporal stability of the PEC micelles at physiological ionic strength was monitored (Figure 2c). GFP(-24) and GFP(-30), which formed PEC micelles with a critical salt concentration greater than $150 \mathrm{mM} \mathrm{NaCl}$, remained colloidally stable for 7 days. The less negatively charged variant, GFP(-18), which had a critical salt concentration near physiological ionic strength, formed micelles that swelled slightly over time and over the course of the week had a slight decrease in scattering intensity of the solution, as monitored by the derived count rate. Conversely, the tagged GFP variants rapidly swelled $(\sim 2-8 \mathrm{~h})$ in the presence of $150 \mathrm{mM} \mathrm{NaCl}$ and demonstrated decreased scattering intensity as evidenced by the decrease in the derived count rate (Supporting Figure S7). To support these DLS results, micelles formed with GFP(-24) and GFP(30) at increased salt concentrations were characterized by TEM (Figure 2d, Supporting Tables S4-5). Analysis of the TEM images confirms that there was no increase in the hydrodynamic radius as the salt concentration was increased from $25 \mathrm{mM}$ to $125 \mathrm{mM} \mathrm{NaCl}$. As the salt concentration was further increased to $150 \mathrm{mM}$, swollen PEC micelles were observed for micelles formed with GFP(-24). While this is below the critical salt concentration determined by DLS, the samples were diluted immediately prior to TEM imaging, which may impact the 
observed micelles. As the critical salt concentration by DLS was even higher for GFP(-30) micelles, a similar increase in size was not observed by TEM at $150 \mathrm{mM} \mathrm{NaCl}$.

\section{GFP delivery with PEC micelles.}

With an understanding of the PEC micelle size and stability at physiological ionic strength, we then proceeded to study the delivery of anionic GFP mutants to Jurkat T cells. We investigated the delivery of the anionic proteins and their PEC micelle counterparts for the variants that formed micelles at or near physiological ionic strength (GFP(-18), GFP(-24), GFP(-30), and tag-GFP(24)). Solutions containing PEC micelles were incubated with Jurkat cells at $37^{\circ} \mathrm{C}$ for $1 \mathrm{~h}$ (final macromolecule concentrations of 0.05 and 0.1 $\mathrm{mg} \mathrm{mL}^{-1}$ ). After washing, the cell fluorescence was quantified by flow cytometry (Figure 4, Supporting Figures S9-16). In addition to the GFP loaded micelles, the delivery of a cationic variant, containing a poly(arginine) tag (GFP(+6)-6R), was evaluated for comparison. It has previously been demonstrated that arginine rich peptide fusions promote cellular internalization of fluorescent proteins..$^{50,51}$

The flow cytometry data indicated a clear relationship between cytotoxicity and micelle stability. Exposure to the GFP(+6)-6R positive control, as well as the free protein solutions of the anionic GFPs, resulted in a minimal decrease in cell viability (Figure 4a, Supporting Table S6). Treatment with the free polymer, however,
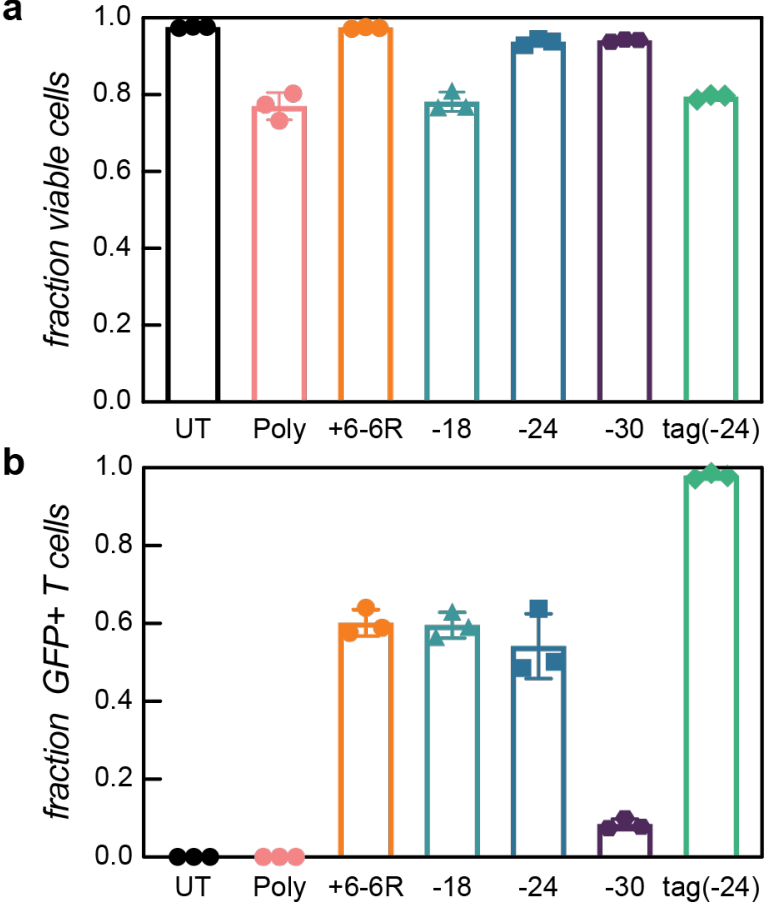

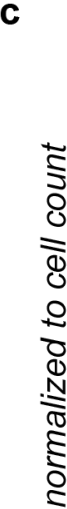

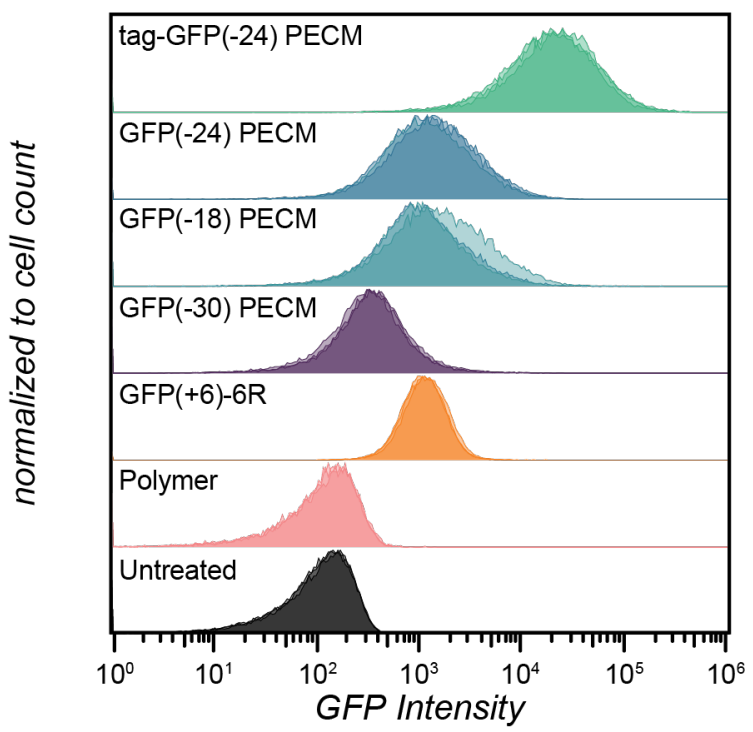

Figure 4. Effect of protein charge on the deliveyr of GFP via PECMs. All samples treated with Sytox Aadvanced. Poly and +6-6R samples contain $0.05 \mathrm{mg} \mathrm{mL}^{-1}$ of polymer and protein, respectively. PECM samples contain $\mathrm{mg} \mathrm{mL}^{-1}$ of polymer and protein each. (a) Ungated fraction of Sytox PECM Jurkat $T$ cells. The mean, standard deviation, and individual data points of 3 biological replicates are shown. (b) Fraction of GFP ${ }^{+}$PECM treated Jurkat $T$ cells, gated for $T$ cells. The mean, standard deviation, and individual data points of 3 biological replicates are shown. (c) Histograms of GFP intensity of PECM treated Jurkat T cells, gated for T cells. Shown are overlayed histograms for 3 biological replicates. 
resulted in approximately a $20 \%$ decrease in cell viability (Figure 4a, Supporting Table S6). For the micelles formed with tag-GFP(-24) and GFP(-18), which have a critical salt concentration below $150 \mathrm{mM}$, cell viability saw a similar 20\% decrease upon incubation with micelles. GFP(24) and GFP(-30) micelles, which were stable at physiological ionic strength, resulted in modest decreases in viability ( $8 \%$ and $5 \%$, respectively, Figure $4 a$ ). We hypothesize that the decrease in cell viability predominantly correlates with the concentration of free polymer in solution, which increases as micelle stability decreases. Importantly though, the interest in using polycations for nucleic acid delivery has spurred the study of cytocompatible polycations. ${ }^{52}$ Altering the polycation in this system could, therefore, further reduce the observed cytotoxicity.

GFP delivery efficiency to Jurkat cells was quantified by flow cytometry (Figure 4b,c Supporting Table S6). Cellular uptake of GFP(+6)-6R was 10-fold greater than uptake of any of the anionic proteins variants, demonstrating the challenge of delivering negatively charged proteins (Supporting Figures S10-16). Critically, complexation of the anionic proteins with the cationic block copolymer resulted in successful internalization of the GFP variants. PEC micelles formed with the most anionic protein, GFP(-30), resulted in the least efficient cellular uptake, with only a 5-fold increase over the free protein solution (Figure 4c). Cellular delivery efficiency further increased for both GFP(-24) and GFP(-18) PEC micelles, with uptake similar to the cationic GFP control (Figure 4b,c). GFP(-18) delivery by micelles was also modestly dependent on the protein and polymer mixing ratio, with the higher positive charge fraction $\left(f^{+}=0.85\right)$ showing a $50 \%$ increase relative to a mixing ratio with more of the anionic protein $\left(f^{+}=0.79\right)$ (Supporting Figures S11 and S16). We hypothesize that these changes in cellular delivery correlate with the scattering intensity of the micelles at low salt, which would be indicative of more particles scattering in solution (Supporting Figure S5). Tag-GFP(-24) PEC micelles resulted in the most efficient cellular uptake, with a 100 -fold increase over anionic proteins alone and a 10-fold increase compared to the cationic GFP(+6)-6R control (Figure 4c). These combined results reveal a convincing inverse relationship between micelle stability and delivery efficiency. We initially hypothesized that a PEC micelle $<200 \mathrm{~nm}$ in diameter at physiological ionic strength would result in the most efficient GFP delivery. However, the most salt stable micelles, formed with the GFP(-30) mutant, have a hydrodynamic diameter less than $200 \mathrm{~nm}$ at physiological ionic strength but delivered the least efficiently. We therefore propose that smaller protein/polymer complexes are more efficiently delivered than intact micelles. While PEC micelles formed with 


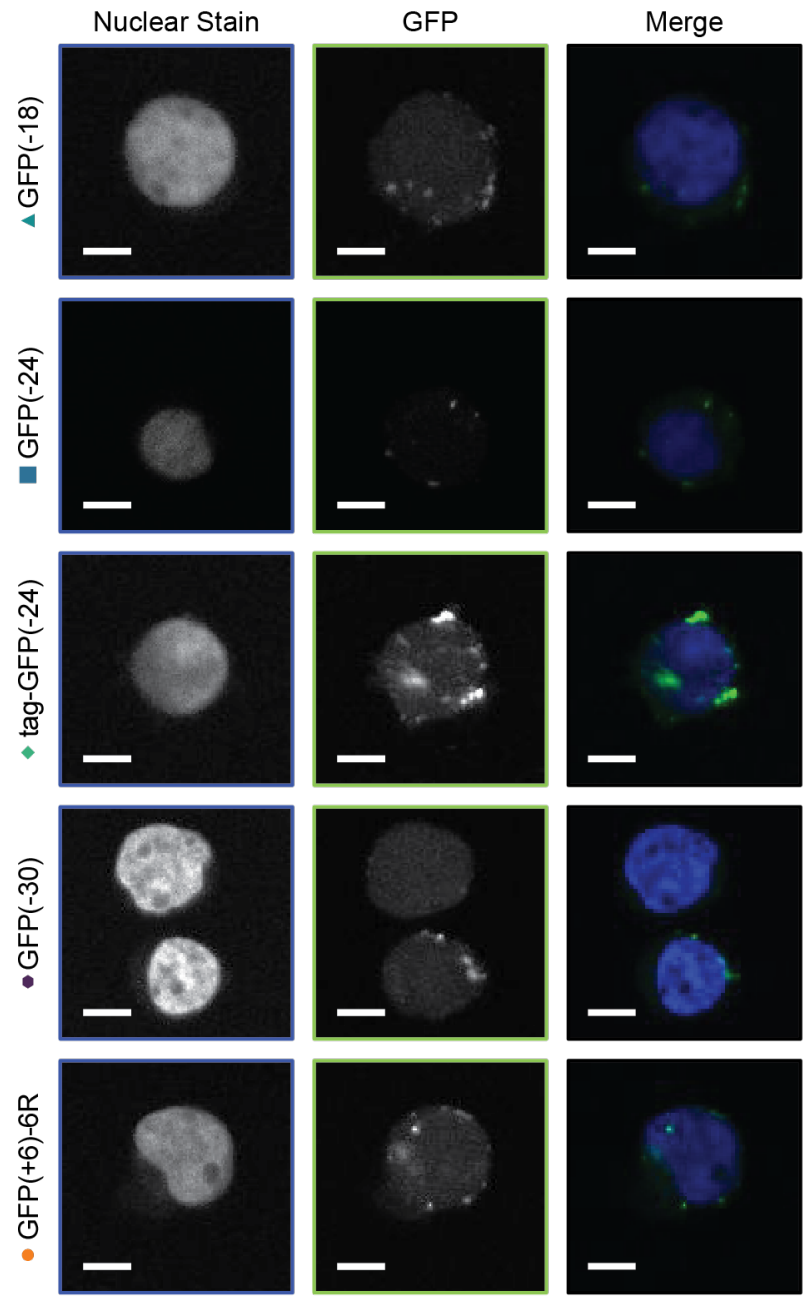

Figure 5. Confocal images of Jurkat T cells. Cells were incubated with GFP containing micelles for $1 \mathrm{~h}$ and then washed $3 \mathrm{x}$ with DPBS. Cells were fixed with $4 \%$ formaldehyde in DPBS and stained with NucBlue for $15 \mathrm{~min}$. Scale bar $=5 \mu \mathrm{m}$
tag-GFP(-24) were not stable in solution at physiological ionic strength, the anionic protein likely still interacts with the polymer at this ionic strength forming small complexes, as evidenced by DLS. These smaller complexes, particularly if they contain excess cationic charge, would more preferentially interact with the cellular membrane and be more effectively endocytosed. While not as drastic for the isotropic GFP variants with intermediate charge, the modest PEC micelle stability near physiological ionic strength suggests that these proteins may similarly have preferential uptake through smaller protein/polymer complexes.

Finally, the uptake of GFP was corroborated by confocal microscopy, which correlated with the flow cytometry analysis (Figure 5, Supporting Figure S17). For all GFP variants, cellular internalization was observed after $1 \mathrm{~h}$ of

incubation with PEC micelles or cationic protein. The cationic poly(arginine) tag protein was observed to co-localize with a dye that stains lysosomes (LysoTracker), consistent with internalization via endocytosis (Supporting Figure S17).9,53,54 GFP delivered by PEC micelles was observed to be associated with the perimeter of the cell and primarily localized in puncta, the latter also consistent with internalization via endocytosis. ${ }^{55}$

\section{Conclusions}

A panel of anionic supercharged GFPs and a cationic diblock copolymer were developed to study the impact of protein charge and charge distribution on PEC micelle formation for intracellular protein delivery. All of the proteins formed PEC micelles with the diblock copolymer at several mixing ratios. Supercharging the model protein with a polyionic tag produced PEC 
micelles with a liquid-like core, whereas isotropically supercharging GFP resulted in PEC micelles with a less dynamic core in the absence of salt. Increasing the solution ionic strength, resulted in isotropic PEC micelles with a more dynamic core as evidenced by a smaller hydrodynamic radius and lower polydispersity index. All mutants produced PEC micelles that remained colloidally stable for 1 week at low ionic strength. Supercharging GFP with an anionic tag produced PEC micelles with lower critical salt concentrations, suggesting that polymers in the PEC micelle core interact differently with the protein tag and the globular domain. The critical salt concentration of the isotropic variants increased with decreasing protein net charge, which is consistent with prior work showing a monotonic increase as a function of protein charge. The

intracellular delivery of the PEC micelles was compared to a GFP control with a cell-penetrating poly(arginine) peptide. Complexation of the anionic proteins with the cationic block copolymer resulted in internalization of the GFP variants at least as efficiently as the cationic protein control. An inverse relationship was established between GFP delivery efficiency and PEC micelle salt stability, indicating that smaller protein/polymer complexes are more efficiently delivered to cells. This highlights the importance of characterizing both the solution structure and activity of polyelectrolyte complexes as they are not inherently directly correlated. These results also indicate the need to continue developing non-toxic cationic delivery agents as well as additional approaches to facilitate the early endosomal escape of endocytosed proteins.

\section{Author Contributions}

R.A.K. and A.C.O. are jointly responsible for the conception, design, analysis, and writing of the studies described herein.

\section{Conflicts of Interest}

There are no conflicts to declare.

\section{Acknowledgements}

The authors acknowledge the Fu Foundation School of Engineering and Applied Sciences at Columbia University of funding. 


\section{References}

(1) Dimitrov, D. S. Therapeutic Proteins. In Therapeutic Proteins: Methods and Protocols; Voynov, V., Caravella, J. A., Eds.; Methods in Molecular Biology; Humana Press: Totowa, NJ, 2012; pp 1-26. https://doi.org/10.1007/978-1-61779-921-1_1.

(2) Pisal, D. S.; Kosloski, M. P.; Balu-lyer, S. V. Delivery of Therapeutic Proteins. Journal of Pharmaceutical Sciences 2010, 99 (6), 2557-2575. https://doi.org/10.1002/jps.22054.

(3) Fu, A.; Tang, R.; Hardie, J.; Farkas, M. E.; Rotello, V. M. Promises and Pitfalls of Intracellular Delivery of Proteins. Bioconjugate Chem. 2014, 25 (9), 1602-1608. https://doi.org/10.1021/bc500320j.

(4) Leader, B.; Baca, Q. J.; Golan, D. E. Protein Therapeutics: A Summary and Pharmacological Classification. Nat Rev Drug Discov 2008, 7 (1), 21-39. https://doi.org/10.1038/nrd2399.

(5) Horn, J. M.; Obermeyer, A. C. Genetic and Covalent Protein Modification Strategies to Facilitate Intracellular Delivery. Biomacromolecules 2021, 22 (12), 4883-4904. https://doi.org/10.1021/acs.biomac.1c00745.

(6) Mix, K. A.; Lomax, J. E.; Raines, R. T. Cytosolic Delivery of Proteins by Bioreversible Esterification. J. Am. Chem. Soc. 2017, 139 (41), 14396-14398. https://doi.org/10.1021/jacs.7b06597.

(7) Schmidt, S.; Adjobo-Hermans, M. J. W.; Kohze, R.; Enderle, T.; Brock, R.; Milletti, F. Identification of Short Hydrophobic Cell-Penetrating Peptides for Cytosolic Peptide Delivery by Rational Design. Bioconjug Chem 2017, 28 (2), 382-389. https://doi.org/10.1021/acs.bioconjchem.6b00535.

(8) Ramakrishna, S.; Kwaku Dad, A.-B.; Beloor, J.; Gopalappa, R.; Lee, S.-K.; Kim, H. Gene Disruption by Cell-Penetrating Peptide-Mediated Delivery of Cas9 Protein and Guide RNA. Genome Res 2014, 24 (6), 1020-1027. https://doi.org/10.1101/gr.171264.113.

(9) Hu, J.; Lou, Y.; Wu, F. Improved Intracellular Delivery of Polyarginine Peptides with Cargoes. J. Phys. Chem. B 2019, 123 (12), 2636-2644.

https://doi.org/10.1021/acs.jpcb.8b10483.

(10) Copolovici, D. M.; Langel, K.; Eriste, E.; Langel, Ü. Cell-Penetrating Peptides: Design, Synthesis, and Applications. ACS Nano 2014, 8 (3), 1972-1994. https://doi.org/10.1021/nn4057269.

(11) Ye, J.; Liu, E.; Yu, Z.; Pei, X.; Chen, S.; Zhang, P.; Shin, M.-C.; Gong, J.; He, H.; Yang, V. C. CPP-Assisted Intracellular Drug Delivery, What Is Next? Int J Mol Sci 2016, 17 (11), E1892. https://doi.org/10.3390/ijms17111892.

(12) Cronican, J. J.; Beier, K. T.; Davis, T. N.; Tseng, J.-C.; Li, W.; Thompson, D. B.; Shih, A. F.; May, E. M.; Cepko, C. L.; Kung, A. L.; Zhou, Q.; Liu, D. R. A Class of Human Proteins That 
Deliver Functional Proteins Into Mammalian Cells In Vitro and In Vivo. Chem Biol 2011, 18 (7), 833-838. https://doi.org/10.1016/j.chembiol.2011.07.003.

(13) Cronican, J. J.; Thompson, D. B.; Beier, K. T.; McNaughton, B. R.; Cepko, C. L.; Liu, D. R. Potent Delivery of Functional Proteins into Mammalian Cells in Vitro and in Vivo Using a Supercharged Protein. ACS Chem. Biol. 2010, 5 (8), 747-752. https://doi.org/10.1021/cb1001153.

(14) Ma, Y.; Poole, K.; Goyette, J.; Gaus, K. Introducing Membrane Charge and Membrane Potential to T Cell Signaling. Frontiers in Immunology 2017, 8, 1513.

https://doi.org/10.3389/fimmu.2017.01513.

(15) Scaletti, F.; Hardie, J.; Lee, Y.-W.; Luther, D. C.; Ray, M.; Rotello, V. M. Protein Delivery into Cells Using Inorganic Nanoparticle-Protein Supramolecular Assemblies. Chem. Soc. Rev. 2018, 47 (10), 3421-3432. https://doi.org/10.1039/C8CS00008E.

(16) Herrera Estrada, L. P.; Champion, J. A. Protein Nanoparticles for Therapeutic Protein Delivery. Biomater Sci 2015, 3 (6), 787-799. https://doi.org/10.1039/c5bm00052a.

(17) Gu, Z.; Biswas, A.; Zhao, M.; Tang, Y. Tailoring Nanocarriers for Intracellular Protein Delivery. Chem. Soc. Rev. 2011, 40 (7), 3638-3655. https://doi.org/10.1039/C0CS00227E.

(18) Biswas, A.; Joo, K.-I.; Liu, J.; Zhao, M.; Fan, G.; Wang, P.; Gu, Z.; Tang, Y. Endoprotease-Mediated Intracellular Protein Delivery Using Nanocapsules. ACS Nano 2011, 5 (2), 1385-1394. https://doi.org/10.1021/nn1031005.

(19) Lv, J.; Tan, E.; Wang, Y.; Fan, Q.; Yu, J.; Cheng, Y. Tailoring Guanidyl-Rich Polymers for Efficient Cytosolic Protein Delivery. Journal of Controlled Release 2020, 320, 412-420. https://doi.org/10.1016/j.jconrel.2020.01.056.

(20) Van Bruggen, C.; Hexum, J. K.; Tan, Z.; Dalal, R. J.; Reineke, T. M. Nonviral Gene Delivery with Cationic Glycopolymers. Acc. Chem. Res. 2019, 52 (5), 1347-1358. https://doi.org/10.1021/acs.accounts.8b00665.

(21) Chatin, B.; Mével, M.; Devallière, J.; Dallet, L.; Haudebourg, T.; Peuziat, P.; Colombani, T.; Berchel, M.; Lambert, O.; Edelman, A.; Pitard, B. Liposome-Based Formulation for Intracellular Delivery of Functional Proteins. Molecular Therapy - Nucleic Acids 2015, 4. https://doi.org/10.1038/mtna.2015.17.

(22) Wang, A.; Yang, T.; Fan, W.; Yang, Y.; Zhu, Q.; Guo, S.; Zhu, C.; Yuan, Y.; Zhang, T.; Gan, Y. Protein Corona Liposomes Achieve Efficient Oral Insulin Delivery by Overcoming Mucus and Epithelial Barriers. Adv Healthc Mater 2019, 8 (12), e1801123. https://doi.org/10.1002/adhm.201801123.

(23) Zuris, J. A.; Thompson, D. B.; Shu, Y.; Guilinger, J. P.; Bessen, J. L.; Hu, J. H.; Maeder, M. L.; Joung, J. K.; Chen, Z.-Y.; Liu, D. R. Cationic Lipid-Mediated Delivery of Proteins Enables Efficient Protein-Based Genome Editing in Vitro and in Vivo. Nature Biotechnology 2015, 33 (1), 73-80. https://doi.org/10.1038/nbt.3081. 
(24) Harada, A.; Kataoka, K. Novel Polyion Complex Micelles Entrapping Enzyme Molecules in the Core: Preparation of Narrowly-Distributed Micelles from Lysozyme and Poly(Ethylene Glycol)-Poly(Aspartic Acid) Block Copolymer in Aqueous Medium. Macromolecules 1998, 31 (2), 288-294. https://doi.org/10.1021/ma971277v.

(25) Harada, A.; Kataoka, K. Formation of Polyion Complex Micelles in an Aqueous Milieu from a Pair of Oppositely-Charged Block Copolymers with Poly(Ethylene Glycol) Segments. Macromolecules 1995, 28 (15), 5294-5299. https://doi.org/10.1021/ma00119a019.

(26) Harada, A.; Kataoka, K. Novel Polyion Complex Micelles Entrapping Enzyme Molecules in the Core. 2. Characterization of the Micelles Prepared at Nonstoichiometric Mixing Ratios. Langmuir 1999, 15 (12), 4208-4212. https://doi.org/10.1021/la981087t.

(27) Jaturanpinyo, M.; Harada, A.; Yuan, X.; Kataoka, K. Preparation of Bionanoreactor Based on Core-Shell Structured Polyion Complex Micelles Entrapping Trypsin in the Core Cross-Linked with Glutaraldehyde. Bioconjugate Chem. 2004, 15 (2), 344-348. https://doi.org/10.1021/bc034149m.

(28) Magana, J. R.; Sproncken, C. C. M.; Voets, I. K. On Complex Coacervate Core Micelles: Structure-Function Perspectives. Polymers 2020, 12 (9), 1953. https://doi.org/10.3390/polym12091953.

(29) Lee, Y.; Ishii, T.; Cabral, H.; Kim, H. J.; Seo, J.-H.; Nishiyama, N.; Oshima, H.; Osada, K.; Kataoka, K. Charge-Conversional Polyionic Complex Micelles-Efficient Nanocarriers for Protein Delivery into Cytoplasm. Angewandte Chemie International Edition 2009, 48 (29), 5309-5312. https://doi.org/10.1002/anie.200900064.

(30) Iwashita, K.; Handa, A.; Shiraki, K. Coacervates and Coaggregates: Liquid-Liquid and Liquid-Solid Phase Transitions by Native and Unfolded Protein Complexes. International Journal of Biological Macromolecules 2018, 120, 10-18. https://doi.org/10.1016/j.ijbiomac.2018.08.063.

(31) Yuan, X.; Yamasaki, Y.; Harada, A.; Kataoka, K. Characterization of Stable LysozymeEntrapped Polyion Complex (PIC) Micelles with Crosslinked Core by Glutaraldehyde. Polymer 2005, 46 (18), 7749-7758. https://doi.org/10.1016/j.polymer.2005.02.121.

(32) Lindhoud, S.; de Vries, R.; Norde, W.; Stuart, M. A. C. Structure and Stability of Complex Coacervate Core Micelles with Lysozyme. Biomacromolecules 2007, 8 (7), 22192227. https://doi.org/10.1021/bm0700688.

(33) Lindhoud, S.; Vries, R. de; Schweins, R.; Stuart, M. A. C.; Norde, W. Salt-Induced Release of Lipase from Polyelectrolyte Complex Micelles. Soft Matter 2008, 5 (1), 242-250. https://doi.org/10.1039/B811640G.

(34) Obermeyer, A. C.; Mills, C. E.; Dong, X.-H.; Flores, R. J.; Olsen, B. D. Complex Coacervation of Supercharged Proteins with Polyelectrolytes. Soft Matter 2016, 12 (15), 35703581. https://doi.org/10.1039/C6SM00002A. 
(35) Lee, Y.; Ishii, T.; Kim, H. J.; Nishiyama, N.; Hayakawa, Y.; Itaka, K.; Kataoka, K. Efficient Delivery of Bioactive Antibodies into the Cytoplasm of Living Cells by ChargeConversional Polyion Complex Micelles. Angewandte Chemie International Edition 2010, 49 (14), 2552-2555. https://doi.org/10.1002/anie.200905264.

(36) Kim, A.; Miura, Y.; Ishii, T.; Mutaf, O. F.; Nishiyama, N.; Cabral, H.; Kataoka, K. Intracellular Delivery of Charge-Converted Monoclonal Antibodies by Combinatorial Design of Block/Homo Polyion Complex Micelles. Biomacromolecules 2016, 17 (2), 446-453. https://doi.org/10.1021/acs.biomac.5b01335.

(37) A. Kapelner, R.; C. Obermeyer, A. Ionic Polypeptide Tags for Protein Phase Separation. Chemical Science 2019, 10 (9), 2700-2707. https://doi.org/10.1039/C8SC04253E.

(38) Ainis, W. N.; Boire, A.; Solé-Jamault, V.; Nicolas, A.; Bouhallab, S.; Ipsen, R. Contrasting Assemblies of Oppositely Charged Proteins. Langmuir 2019, 35 (30), 9923-9933. https://doi.org/10.1021/acs.langmuir.9b01046.

(39) Simon, A. J.; Zhou, Y.; Ramasubramani, V.; Glaser, J.; Pothukuchy, A.; Gollihar, J.; Gerberich, J. C.; Leggere, J. C.; Morrow, B. R.; Jung, C.; Glotzer, S. C.; Taylor, D. W.; Ellington, A. D. Supercharging Enables Organized Assembly of Synthetic Biomolecules. Nature Chem 2019, 11 (3), 204-212. https://doi.org/10.1038/s41557-018-0196-3.

(40) Kim, S.; Sureka, H. V.; Kayitmazer, A. B.; Wang, G.; Swan, J. W.; Olsen, B. D. Effect of Protein Surface Charge Distribution on Protein-Polyelectrolyte Complexation.

Biomacromolecules 2020, 21 (8), 3026-3037. https://doi.org/10.1021/acs.biomac.0c00346.

(41) van Lente, J. J.; Claessens, M. M. A. E.; Lindhoud, S. Charge-Based Separation of Proteins Using Polyelectrolyte Complexes as Models for Membraneless Organelles. Biomacromolecules 2019, 20 (10), 3696-3703. https://doi.org/10.1021/acs.biomac.9b00701.

(42) McTigue, W. C. B.; Perry, S. L. Design Rules for Encapsulating Proteins into Complex Coacervates. Soft Matter 2019, 15 (15), 3089-3103. https://doi.org/10.1039/C9SM00372J.

(43) Pédelacq, J.-D.; Cabantous, S.; Tran, T.; Terwilliger, T. C.; Waldo, G. S. Engineering and Characterization of a Superfolder Green Fluorescent Protein. Nat Biotech 2006, 24 (1), 79-88. https://doi.org/10.1038/nbt1172.

(44) Teo, J.; McCarroll, J. A.; Boyer, C.; Youkhana, J.; Sagnella, S. M.; Duong, H. T. T.; Liu, J.; Sharbeen, G.; Goldstein, D.; Davis, T. P.; Kavallaris, M.; Phillips, P. A. A Rationally Optimized Nanoparticle System for the Delivery of RNA Interference Therapeutics into Pancreatic Tumors in Vivo. Biomacromolecules 2016, 17 (7), 2337-2351. https://doi.org/10.1021/acs.biomac.6b00185.

(45) Ozer, I.; Tomak, A.; Zareie, H. M.; Baran, Y.; Bulmus, V. Effect of Molecular Architecture on Cell Interactions and Stealth Properties of PEG. Biomacromolecules 2017, 18 (9), 2699-2710. https://doi.org/10.1021/acs.biomac.7b00443. 
(46) Zheng, Y.; Won, Y.-Y.; Bates, F. S.; Davis, H. T.; Scriven, L. E.; Talmon, Y. Directly Resolved Core-Corona Structure of Block Copolymer Micelles by Cryo-Transmission Electron Microscopy. J. Phys. Chem. B 1999, 103 (47), 10331-10334.

https://doi.org/10.1021/jp9923264.

(47) Sprouse, D.; Jiang, Y.; Laaser, J. E.; Lodge, T. P.; Reineke, T. M. Tuning Cationic Block Copolymer Micelle Size by PH and lonic Strength. Biomacromolecules 2016, 17 (9), 28492859. https://doi.org/10.1021/acs.biomac.6b00654.

(48) Shah, S.; Leon, L. Structural Dynamics, Phase Behavior, and Applications of Polyelectrolyte Complex Micelles. Current Opinion in Colloid \& Interface Science 2021, 53, 101424. https://doi.org/10.1016/j.cocis.2021.101424.

(49) Amann, M.; Diget, J. S.; Lyngsø, J.; Pedersen, J. S.; Narayanan, T.; Lund, R. Kinetic Pathways for Polyelectrolyte Coacervate Micelle Formation Revealed by Time-Resolved Synchrotron SAXS. Macromolecules 2019, 52 (21), 8227-8237. https://doi.org/10.1021/acs.macromol.9b01072.

(50) Chang, M.; Chou, J.-C.; Lee, H.-J. Cellular Internalization of Fluorescent Proteins via Arginine-Rich Intracellular Delivery Peptide in Plant Cells. Plant and Cell Physiology 2005, 46 (3), 482-488. https://doi.org/10.1093/pcp/pci046.

(51) Fuchs, S. M.; Raines, R. T. Polyarginine as a Multifunctional Fusion Tag. Protein Sci 2005, 14 (6), 1538-1544. https://doi.org/10.1110/ps.051393805.

(52) Kumar, R.; Le, N.; Tan, Z.; Brown, M. E.; Jiang, S.; Reineke, T. M. Efficient PolymerMediated Delivery of Gene-Editing Ribonucleoprotein Payloads through Combinatorial Design, Parallelized Experimentation, and Machine Learning. ACS Nano 2020, 14 (12), 17626-17639. https://doi.org/10.1021/acsnano.0c08549.

(53) Allen, J. K.; Brock, D. J.; Kondow-McConaghy, H. M.; Pellois, J.-P. Efficient Delivery of Macromolecules into Human Cells by Improving the Endosomal Escape Activity of CellPenetrating Peptides: Lessons Learned from DfTAT and Its Analogs. Biomolecules 2018, 8 (3), E50. https://doi.org/10.3390/biom8030050.

(54) Erazo-Oliveras, A.; Muthukrishnan, N.; Baker, R.; Wang, T.-Y.; Pellois, J.-P. Improving the Endosomal Escape of Cell-Penetrating Peptides and Their Cargos: Strategies and Challenges. Pharmaceuticals (Basel) 2012, 5 (11), 1177-1209. https://doi.org/10.3390/ph5111177.

(55) Luther, D. C.; Jeon, T.; Goswami, R.; Nagaraj, H.; Kim, D.; Lee, Y.-W.; Rotello, V. M. Protein Delivery: If Your GFP (or Other Small Protein) Is in the Cytosol, It Will Also Be in the Nucleus. Bioconjugate Chem. 2021, 32 (5). https://doi.org/10.1021/acs.bioconjchem.1c00103. 